V.M. Mikhailov, K.V. Chunikhin

\title{
ON ELECTROSTATIC ANALOGY OF MAGNETOSTATIC FIELD IN INHOMOGENEOUS MAGNETIZED MEDIUM
}

\begin{abstract}
Purpose. The application in electrostatic analogy of magnetostatics for inhomogeneous magnetized media of two known magnetization models. Methodology. A comparison of basic equations and formulas of electrostatic and magnetostatic field in immoveable isotropic inhomogeneous polarized medium for dipole model and the magnetization model by molecular currents is made. The value-analogues for dipole model of magnetization are established. Results. We have shown that the using of dipole model of magnetization is correct. There is not even formal analogy with electrostatic field in the case of using the magnetization model by molecular currents. The relation between the magnetizations for various models is obtained. It allows us to justify the using magnetization by molecular currents in electrostatic analogy. Originality. The magnetization for dipole model is introduced and the possibility of using magnetization by molecular currents in the formulas for calculating potential magnetostatic field in magnetized medium is substantiated. Practical value. The results allow to obtain correct formulation and solution of the problem of magnetostatic field calculation in inhomogeneous magnetized medium. References 5, tables 1.
\end{abstract}

Key words: magnetostatics, magnetized medium, electrostatic analogy, dipole model, magnetization by molecular currents.

Сделан анализ применения электростатической аналогии в магнитостатике неоднородных намагничивающихся сред на основе дипольной модели и модели намагничивания молекулярными токами. Показано, что корректным является использование дипольной модели намагничивания. Получено соотночение между намагниченностями для различных моделей, поясняющее применение в электростатической аналогии намагниченности молекулярными токами. Библ. 5, табл. 1.

Ключевые слова: магнитостатика, намагничивающаяся среда, электростатическая аналогия, дипольная модель, молекулярный ток намагничивания, намагниченность.

Introduction. The use of fictitious magnetic charges for the calculation of magnetostatic fields in magnetized media is associated with the development of ideas about the nature of magnetic phenomena and is based on the electrostatic analogy of the polarization of dielectrics and magnets [1]. This analogy is based on the formation of magnetic dipoles, like electric dipoles, but consisting of two point fictitious magnetic charges (hereinafter - the dipole model of magnetization). The electrostatic analogy was used, in particular, by G.A. Greenberg [2] and K. Simonyi [3].

Subsequently it turned out that magnetization occurs due to the flow of molecular (microscopic) currents inside the magnets (such a model will be called the magnetization model by molecular currents), and the electrostatic analogy is far from the nature of magnetism $[1,4]$. Despite the formal nature, the electrostatic analogy, when correctly used, is very effective [1-4].

To different models of magnetization we put in correspondence various magnetizations: $\vec{J}^{e}-$ magnetization by dipoles; $\vec{J}$ - magnetization by molecular currents. In some books and articles, the authors describe the magnetostatic field of magnetized bodies with the help of the scalar potential $\varphi_{m}$ which corresponds to the dipole model, and the magnetization $\vec{J}$ is used in the formulas for determining $\varphi_{m}$. A possible reason for this is the analogy proposed in the textbook [1] between the polarization of the dielectric $\vec{P}$ and $\mu_{0} \vec{J}\left(\mu_{0}\right.$ is the magnetic constant), but the latter requires a theoretical justification.

The relevance of this paper is that the application of the electrostatic analogy for the formulation and testing of numerical algorithms for solving magnetostatic problems of magnetized bodies leads to difficulties associated with a lack of clarity in well-known publications.
The goal of the work is analysis of the correctness of the application in the electrostatic analogy of magnetostatics of inhomogeneous magnetizing media of two known models of magnetization.

Basic equations and formulas of electrostatic analogy. We consider the electrostatic and magnetostatic field in a stationary isotropic inhomogeneous polarized medium. The basic equations of these fields and formulas are presented in Table 1 [1-4]. The analogues in the case of the dipole model of magnetization are: $\vec{E}$ and $\vec{H}, \vec{D}$ and $\vec{B}$ are the intensities, as well as the induction of the electric and magnetic fields; and; $\vec{P}$ and $\vec{J}^{e} ; \rho_{e}$ and $\rho_{m}$ are the volume densities of the polarization electric and fictitious magnetic charges; $\rho_{e}^{s t}$ and $\rho_{m}^{s t}$ are the volume densities of external [2] electric and fictitious magnetic charges; $\varepsilon_{0}$ и $\mu_{0}, \varepsilon_{0}$ is the electric constant; $\vec{p}$ and $\vec{p}_{m e}$ are the moments of electric and magnetic dipoles; $\pm q$ and $\pm m$ are the point electric and fictitious magnetic charges located in the dipoles at distance $l$. In the formulas for determining $\vec{P}$ and $\vec{J}^{e}$ the quantity $\Delta V$ is a sufficiently small volume of the polarizable medium, over which the sums of the corresponding dipole moments are averaged. The field of magnetic dipoles is potential, its scalar potential $\varphi_{m}$ is analogous to the potential of the electrostatic field $\varphi$. A consequence of the formality of the analogy in question is that magnetic flux density $\vec{B}$ becomes an auxiliary vector. In the formulas for calculating $\varphi_{m}$ and $\varphi$, the following designations are accepted: in the case of dipoles distributed in a volume $V$, the quantity $d V_{M}$ is the elementary volume centered at the point $M \in V$; in the case of surface fictitious magnetic charges distributed on the boundary surface $S$, the quantity $d S_{M}$ is the elementary area centered at the point $M \in S ; r_{M Q}$ is the distance between the point with the current coordinates $M$ and the observation point $Q$.

(C) V.M. Mikhailov, K.V. Chunikhin 
Basic equations and formulas for analogous fields calculation

\begin{tabular}{|c|c|}
\hline Electrostatic field & $\begin{array}{c}\text { Magnetostatic field } \\
\text { (dipole magnetization model) }\end{array}$ \\
\hline $\operatorname{div} \vec{E}=\frac{\rho_{e}}{\varepsilon_{0}}+\frac{\rho_{e}^{s t}}{\varepsilon_{0}}$ & $\operatorname{div} \vec{H}=\frac{\rho_{m}}{\mu_{0}}+\frac{\rho_{m}^{s t}}{\mu_{0}}$ \\
\hline $\operatorname{div} \vec{P}=-\rho_{e}$ & $\operatorname{div} \vec{J}^{e}=-\rho_{m}$ \\
\hline$\vec{D}=\varepsilon_{0} \vec{E}+\vec{P}$ & $\vec{B}=\mu_{0} \vec{H}+\vec{J}^{e}$ \\
\hline $\operatorname{div} \vec{D}=\rho_{e}^{s t}$ & $\operatorname{div} \vec{B}=\rho_{m}^{s t}$ \\
\hline$\vec{P}=\frac{1}{\Delta V} \sum_{\Delta V} \vec{p}$ & $\vec{J}^{e}=\frac{1}{\Delta V} \sum_{\Delta V} \vec{p}_{m e}$ \\
\hline$\vec{p}=q \vec{l}$ & $\vec{p}_{m e}=m \vec{l}$ \\
\hline$\vec{E}=-\operatorname{grad} \varphi$ & $\vec{H}=-\operatorname{grad}_{m}$ \\
\hline$\frac{1}{4 \pi \varepsilon_{0}} \int\left(\vec{P}(M), \operatorname{grad}_{M} \frac{1}{r_{M Q}}\right) d V_{M}$ & $\varphi_{m}(Q)=\frac{1}{4 \pi \mu_{0}} \int \frac{1}{V}\left(\vec{J}^{e}(M), \operatorname{grad}_{M} \frac{1}{r_{M Q}}\right) d V_{M}$ \\
\hline$\varphi(Q)=\frac{1}{4 \pi \varepsilon_{0}} \int_{S} \sigma(M) \frac{1}{r_{M Q}} d S_{M}$ & $\varphi_{m}(Q)=\frac{1}{4 \pi \mu_{0}} \int_{S} \sigma_{m}(M) \frac{1}{r_{M Q}} d S_{M}$ \\
\hline
\end{tabular}

For the interface of two different homogeneous media 1 and 2 in the absence of external sources and assuming that the normal to the boundary is directed to the medium 1, from the equations of the first row of Table 1, 1 the boundary conditions [2] for the electrostatic field follow -

$$
E_{1 n}-E_{2 n}=\frac{\sigma_{e}}{\varepsilon_{0}}
$$

and for the magnetostatic field -

$$
H_{1 n}-H_{2 n}=\frac{\sigma_{m}}{\mu_{0}}
$$

where $\sigma_{e}, \sigma_{m}$ are the surface densities of polarization electric and fictitious magnetic charges at the interface, and

$$
\sigma_{e}=P_{2 n}-P_{1 n}, \sigma_{m}=J_{2 n}^{e}-J_{1 n}^{e} .
$$

In the boundary conditions (1) and (2), as well as in formulas (3), the indices $1 n$ and $2 n$ have normal projections of the corresponding vectors in media 1 and 2 .

Analysis of the application of the model of magnetization by molecular currents. First, let us cite in the same sense order, as for the dipole model (see Table 1), the basic equations and magnetostatic formulas for magnetized media based on the model of magnetization by molecular currents $[1,4]$ :

$$
\begin{gathered}
\operatorname{rot} \vec{B}=\mu_{0}\left(\vec{j}_{m}+\vec{\delta}^{s t}\right) ; \\
\operatorname{rot} \vec{J}=\vec{j}_{m} ; \\
\vec{B}=\mu_{0}(\vec{H}+\vec{J}) ; \\
\operatorname{div} \vec{B}=0 ; \\
\vec{J}=\frac{1}{\Delta V} \sum_{\Delta V} \vec{p}_{m} ; \\
\vec{p}_{m}=i_{m} s \vec{n} ;
\end{gathered}
$$

$$
\begin{gathered}
\vec{B}=\operatorname{rot} \vec{A} ; \\
\vec{A}(Q)=\frac{\mu_{0}}{4 \pi} \int_{V} \vec{j}_{m}(M) \frac{1}{r_{M Q}} d V_{M},
\end{gathered}
$$

where $\vec{j}_{m}$ is the density of molecular magnetizing currents; $\vec{\delta}^{s t}$ is the density of external conductive currents; $\vec{p}_{m}$ is the magnetic moment of the molecular magnetizing current $i_{m} ; s$ is the area of a microscopic circle bounded by the current $i_{m}$ flow path; $\vec{n}$ is the normal to the microscopic circle in its center; $\vec{A}(Q)$ is the vector potential of the magnetic field created by the molecular magnetizing currents.

Comparison of the basic equations and formulas of the electrostatic field of the polarizable media (see Table 1) and the magnetostatic field of the magnetized media. This question is not limited only to the discrepancy between the relations between the vectors $\vec{D}, \vec{E}, \vec{P}$ and $\vec{B}, \vec{H}, \vec{J}$, and the difference between the sources of the fields, the sources of the electrostatic field, dipole-scalar sources, the magnetostatic sources are the currents (vector sources). The magnetic field of molecular currents, in contrast to the field of magnetic dipoles, is vortical, so its vectors and source densities are related to other equations.

Therefore, we can only talk about the use in electrostatic analogy based on the dipole model of magnetization.

On the relationship between magnetizations $\vec{J}^{e}$ and $\vec{J}$. As we already noted in the Introduction, K.M. Polivanov proposed an analogy of $\vec{P}$ and $\mu_{0} \vec{J}$ [1] using the transformation of formula (4) to form

$$
\vec{B}=\mu_{0} \vec{H}+\mu_{0} \vec{J} .
$$


In this case, to the term $\mu_{0} \vec{J}$ is not given a certain physical meaning, which can be guessed. To clarify the latter, we find the relationship between $\vec{J}^{e}$ and $\vec{J}$.

First, let us use the idea of replacing circuits with conduction currents by double magnetic layers [4, 5]. Applying this idea to the contour of the molecular magnetization current $i_{m}$, we arrive at a microscopic homogeneous double layer of fictitious magnetic charges, which is equivalent to a magnetic dipole.

Secondly, we use the condition of equivalence of the magnetic fields of the circuit with the conduction current at large distances from it and a magnetic dipole in a homogeneous medium with absolute magnetic permeability $\mu$ [5]. Applying this condition to the magnetization models under consideration with microscopic sources, we have

$$
\vec{p}_{m e}=\mu_{0} \vec{p}_{m} .
$$

Averaging left and right sides of condition (5) over a sufficiently small volume of the magnetized medium $\Delta V$, we find

$$
\vec{J}^{e}=\mu_{0} \vec{J} .
$$

The relation (6) makes it possible to use magnetization by molecular currents in the dipole model of magnetization and reveals the physical meaning of $\mu_{0} \vec{J}$ in the analogy under discussion.

Conclusions. The dipole magnetization model and the magnetization model by molecular currents have a different physical meaning which results in different definitions of magnetization and mathematical descriptions of the magnetostatic field in a magnetized medium. The field of the dipole model is potential and is described by a scalar potential, the field of molecular currents is vortex and is described by a vector potential. The application of the electrostatic analogy for the calculation of the magnetostatic field of inhomogeneous magnetizing media is correct on the basis of the dipole magnetization model, however in the calculated formulas of the potential field, it is possible to correctly use the magnetization by molecular currents.

\section{REFERENCES}

1. Polivanov K.M. Teoreticheskie osnovy elektrotekhniki, ch. 3. Teoriia elektromagnitnogo polia [Theoretical foundations of electrical engineering, Part 3. Theory of electromagnetic field]. Moscow, Energija Publ., 1969. 352 p. (Rus).

2. Grinberg G.A. Izbrannye voprosy matematicheskoi teorii elektricheskikh $i$ magnitnykh iavlenii [Selected questions of mathematical theory of electric and magnetic phenomena]. Moscow-Leningrad, Acad. of Sci. USSR Publ., 1948. 730 p. (Rus).

3. Simonyi K. Teoreticheskaya elektrotekhnika [Theoretical Electrical Engineering]. Moscow, Mir Publ., 1964. 775 p. (Rus). 4. Tamm I.E. Osnovy teorii elektrichestva [Fundamentals of electricity theory]. Moscow, Nauka Publ., 1954. 620 p. (Rus).

5. Brunov B.Ia., Gol'denberg L.M., Kliatskin I.G., Tseitlin L.A., Teoriia elektromagnitnogo polia [Theory of electromagnetic field]. Moscow-Leningrad, Gosenergoizdat Publ., 1962. 512 p. (Rus).

Received 28.08.2017

V.M. Mikhailov ${ }^{1}$, Doctor of Technical Science, Professor,

K.V. Chunikhin ${ }^{2}$, Postgraduate Student,

${ }^{1}$ National Technical University «Kharkiv Polytechnic Institute»,

2, Kyrpychova Str., Kharkiv, 61002, Ukraine,

phone +380 577076052 ,

e-mail: valery.m.mikhailov@gmail.com

${ }^{2}$ State Institution «Institute of Technical Problems

of Magnetism of the NAS of Ukraine»,

19, Industrialna Str., Kharkiv, 61106, Ukraine, phone +380 572992162 ,

e-mail: kvchunikhin@gmail.com

How to cite this article:

Mikhailov V.M., Chunikhin K.V. On electrostatic analogy of magnetostatic field in inhomogeneous magnetized medium. Electrical engineering \& electromechanics, 2017, no.5, pp. 38-40. doi: 10.20998/2074-272X.2017.5.05. 